\title{
Integrated energy system planning study based on load prediction
}

\author{
Jialin Yang ${ }^{1, \mathrm{a}}$, Zhen $\mathrm{Li}^{1, \mathrm{a}}$, Nan Wang ${ }^{1, \mathrm{a}}$, Pengxiang Zhao ${ }^{1, \mathrm{a}}$, Xichao Zhou ${ }^{1, \mathrm{a}}$, Lin Cong ${ }^{1, \mathrm{a}}$, Lu Xue ${ }^{2, b^{*}}$, Yongli Wang ${ }^{2, \mathrm{~b}}$ \\ ${ }^{1}$ State Grid Integrated Energy Service Group Co., Ltd., Beijing, 100052, China \\ ${ }^{2}$ North China Electric Power University, Beijing, 102206, China
}

\begin{abstract}
The planning of integrated energy system is a very complex multi-objective, multi-constraint, nonlinear, random uncertain hybrid combination optimization problem, its planning and design process should consider not only the system capacity, energy exchange, energy storage, energy and other links between the interdependence, but also the interaction and mixing of cold, hot, electricity and other multi-energy flow, which is essentially a non-deterministic polynomial problem. Based on load prediction technology, combined with scene generation, multi-interconnected energy system modeling and other technologies, around the integrated energy system planning and design, consider the comprehensive evaluation of the whole life cycle, an optimal configuration of the integrated energy system is formed.
\end{abstract}

\section{Introduction}

In recent years, with the increasingly serious problem of environmental pollution, in order to deal with the energy and environmental crisis brought about by the development and utilization of fossil energy, the energy transformation characterized by electricity-centric and large-scale development and utilization of new energy is booming all over the world. Under the promotion of this energy transformation, breaking the existing model of individual planning, individual design and independent operation of the original energy supply system, carrying out the integrated planning design and operation optimization of the social energy system, and finally constructing a regional multi-energy integrated supply system. In the system, large power grid as the "main network" and micro-network, distributed energy and other energy autonomous units as the "local area network" will become the only way to adapt to energy change, and ensure the safety and long-term stability of the energy supply.

Load forecasting is to determine the load data at a specific time in the future based on many factors such as the system's operating characteristics, capacity-increasing decisions, natural conditions and social influences, and meeting certain accuracy requirements. There are many commonly used load forecasting methods [1], the traditional ones are regression analysis method, exponential smoothing method, time series method, Kalman filter method and so on. However, due to the large changes in the load curve and many influencing factors, traditional methods have obvious limitations in forecasting speed and forecasting accuracy, and the actual application effect is relatively poor. With the development of artificial intelligence in recent years, it has been applied in various fields. Among them, intelligent methods such as expert system [2], artificial neural network, fuzzy forecasting method, and wavelet analysis method [3] are widely used. In load forecasting, intelligent algorithms have good advantages. Literature [4] improves the prediction accuracy by applying fuzzy theory to neural networks; Literature [5] reduces the complexity of the original prediction model by combining empirical mode decomposition with deep learning theory, and speeds up The speed of algorithm load forecasting; Literature [6] applies the wheel mixing theory to load forecasting, and uses the ergodicity and uncertainty of chaos to construct the forecasting model, which better reflects the load changes of users; Literature [7] taking the factors that affect load changes into the load forecasting is more targeted; Literature [8] introduces machine learning into the field of load forecasting, which brings new ideas to load forecasting. The above method basically makes predictions based on the original intelligent algorithm. There are relatively few improvements and optimizations to the original algorithm, and the prediction accuracy can be improved.

Due to the widespread coupling between multiple energy sources, load prediction in integrated energy systems will no longer be independent prediction of energy systems, but a joint prediction of multiple loads. Due to the interaction of various related factors in the integrated energy system, the mechanism of action is complex, and the coupling relationship between the relevant factors can not be established accurately by manual analysis or empirical model[9]. In recent years, artificial intelligence technology, represented by machine learning and deep learning, has been widely used in the field of load prediction. Therefore, it can be predicted that the theory of artificial intelligence based on big data will

\footnotetext{
*Corresponding author's e-mail: x11124213797@163.com
} 
be an effective way to solve the multi-load joint prediction in integrated energy systems in the future[10]. At the same time, combining machine learning with optimization theory to achieve self-optimization of machine learning parameters through optimization is also an important research direction to improve the accuracy of predictions..

\section{Cumulative prediction method}

The accuracy of the load forecast of the integrated energy system is directly related to whether the planning and operation are reasonable and effective, so it is an important prerequisite for the planning of the integrated energy system. Traditional energy system load forecasting methods are limited to independent forecasting of various forms of energy. Although they have formed mature theoretical methods and have been verified in practice, they are no longer suitable for future integration due to ignoring the coupling and mutual influence of multiple energies. Load forecasting of the energy system. The difficulties in load forecasting of the integrated energy system are mainly reflected in the following two aspects: There are many influencing factors. Since the integrated energy system comprehensively covers electricity, gas, heat, cold and other energy forms, it will be affected by many factors, such as climate, social economy, regional layout structure, architectural design characteristics, etc., resulting in modeling difficulties prediction. The model is difficult to solve. Multi-dimensional and multi-time scales increase the difficulty of solving the integrated energy system load forecasting model. In recent years, scholars at home and abroad have conducted extensive and in-depth explorations on integrated energy system load forecasting methods, and have achieved many effective results. The cumulative forecasting method will be introduced below.

The cumulative forecasting method is a forecasting technique for fitting polynomial trends proposed by the Italian mathematician P.E. Marchesi in the 1950s. The accumulation of data is the main calculation, and the accumulation process is actually a smoothing of the data sequence. Using the cumulative method constant table, the relationship number can be determined quickly, the calculation becomes very simple, and the method is more practical, so it will be introduced in detail.

Let $t$ be a time variable, with the value $t_{1}<t_{2}<\cdots<t_{n}$, and the corresponding values of the dependent variable $x_{1}, x_{2}, \cdots x_{n}$. The data $x_{t}$ has the following $n$ degree polynomial trend

$$
\mathrm{x}=\alpha_{0}+\alpha_{1} \mathrm{t}+\alpha_{2} \mathrm{t}^{2}+\cdots+\alpha_{\mathrm{n}} \mathrm{t}^{\mathrm{n}}
$$

In the power load problem, $\left\{t_{k}\right\}$ is usually an arithmetic sequence with a tolerance of 1 , such as $1,2, \ldots$, $T$. Consider the general situation here, which is convenient when using the accumulation method constant table later. The same as linear trend (first-order polynomial) prediction, the key is to use the known $\mathrm{T}$ pair data $\left(t_{k}, \mathrm{x}_{k}\right)(k=1,2, \cdots, \mathrm{T})$ to determine the $n+1$ coefficients in the model equation (1).
First define the cumulative sum of each order. For the sequence $x_{k}$, its first-order cumulative sum is $\sum^{(1)} \mathrm{x}_{\mathrm{k}}=\mathrm{x}_{1}+\mathrm{x}_{2}+\cdots+\mathrm{x}_{\mathrm{T}}$;

Second-order cumulative sum:

$\sum^{(2)} \mathrm{x}_{\mathrm{k}}=\mathrm{x}_{1}+\left(\mathrm{x}_{1}+\mathrm{x}_{2}\right)+\cdots+\left(\mathrm{x}_{1}+\mathrm{x}_{2}+\cdots+\mathrm{x}_{\mathrm{T}}\right)$

Third-order cumulative sum:

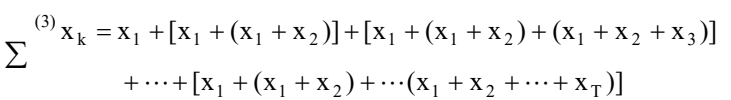

Push in turn. The rule is that the cumulative sum of each order is written as the sum of $T$ terms (as above), and its $K$ term is the sum of the first $K$ terms in the expression of the previous cumulative sum. If expressed by a matrix, write $1=(1,1, \cdots 1)^{\prime}, F=\left(f_{i j}\right)$, where

$$
\mathrm{f}_{\mathrm{ij}}=\left\{\begin{array}{l}
1 \mathrm{i} \geq \mathrm{j} \\
0 \mathrm{i}<\mathrm{j}, \quad i, j=1,2, \cdots T
\end{array}\right.
$$

Substitute $T$ pairs of data into equation (1) to get $T$ equations

$$
\left.\begin{array}{l}
\mathrm{x}_{1}=\alpha_{0}+\alpha_{1} \mathrm{t}_{1}+\alpha_{2} \mathrm{t}_{1}^{2}+\cdots+\alpha_{\mathrm{n}} \mathrm{t}_{1}^{\mathrm{n}} \\
\mathrm{x}_{2}=\alpha_{0}+\alpha_{1} \mathrm{t}_{2}+\alpha_{2} \mathrm{t}_{2}^{2}+\cdots+\alpha_{\mathrm{n}} \mathrm{t}_{2}^{\mathrm{n}} \\
\cdots \cdots \\
\mathrm{x}_{\mathrm{T}}=\alpha_{0}+\alpha_{1} \mathrm{t}_{\mathrm{T}}+\alpha_{2} \mathrm{t}_{\mathrm{T}}^{2}+\cdots+\alpha_{\mathrm{n}} \mathrm{t}_{\mathrm{T}}^{\mathrm{n}}
\end{array}\right\}
$$

Calculate the cumulative sum of order $1 \sim n+1$ on both sides of the equation at the same time to obtain $n+1$ cumulative equations, which can form a system of $n+1$ equations with $\alpha_{0}, \alpha_{1}, \cdots, \alpha_{n}$ as System of $n+1$ equations with unknowns

$\sum^{(1)} x_{k}=\alpha_{0} \sum^{(1)} t_{\mathrm{K}}^{0}+\alpha_{1} \sum^{(1)} t_{k}^{1}+\alpha_{2} \sum^{(1)} t_{k}^{2}+\cdots+\alpha_{n} \sum^{(1)} t_{k}^{n}$

$\sum^{(2)} x_{k}=\alpha_{0} \sum^{(2)} t_{K}^{0}+\alpha_{1} \sum^{(2)} t_{k}^{1}+\alpha_{2} \sum^{(2)} t_{k}^{2}+\cdots+\alpha_{n} \sum^{(2)} t_{k}^{n}$

$\sum^{(n+1)} x_{k}=\alpha_{0} \sum^{(n+1)} t_{K}^{0}+\alpha_{1} \sum^{(n+1)} t_{k}^{1}+\alpha_{2} \sum^{(n+1)} t_{k}^{2}+\cdots+\alpha_{n} \sum^{(n+1)} t_{k}^{n}$

In the above formula:

$\mathrm{t}_{\mathrm{k}}^{0}-$ constant 1 ;

$\sum^{(j)} \mathrm{t}_{\mathrm{k}}^{0}$ _ $\mathrm{J}$-th order cumulative sum of all one sequences;

$\sum^{(j)} t_{k}^{i} \_$J-th order cumulative sum of sequence $\mathrm{t}_{1}^{\mathrm{i}}, \mathrm{t}_{2}^{\mathrm{i}} \cdots, \mathrm{t}_{\mathrm{T}}^{\mathrm{i}}, \mathrm{j}=1,2, \cdots, \mathrm{n}$.

In order to express and solve convenient, remember

$$
\begin{aligned}
& \mathrm{c}=\left\{\begin{array}{ccccc}
\sum^{(1)} \mathrm{t}_{\mathrm{k}}^{0} & \sum^{(1)} \mathrm{t}_{\mathrm{k}}^{1} & \sum^{(1)} \mathrm{t}_{\mathrm{k}}^{2} & \cdots & \sum^{(2)} \mathrm{t}_{\mathrm{k}}^{\mathrm{n}} \\
\sum^{(2)} \mathrm{t}_{\mathrm{k}}^{0} & \sum^{(2)} \mathrm{t}_{\mathrm{k}}^{1} & \sum^{(2)} \mathrm{t}_{\mathrm{k}}^{2} & \cdots & \sum^{(2)} \mathrm{t}_{\mathrm{k}}^{\mathrm{n}} \\
\cdots & \ldots & \cdots & \cdots & \cdots \\
\sum^{(\mathrm{n}+1)} \mathrm{t}_{\mathrm{k}}^{0} & \sum^{(\mathrm{n}+1)} \mathrm{t}_{\mathrm{k}}^{1} & \sum^{(\mathrm{n}+1)} \mathrm{t}_{\mathrm{k}}^{2} & \cdots & \sum^{(\mathrm{n}+1)} \mathrm{t}_{\mathrm{k}}^{\mathrm{n}}
\end{array}\right\} \\
& \mathrm{a}=\left\{\begin{array}{c}
\mathrm{a}_{0} \\
\mathrm{a}_{1} \\
\vdots \\
\mathrm{a}_{\mathrm{n}}
\end{array}\right\} \mathrm{b}=\left\{\begin{array}{c}
\sum^{(1)} \mathrm{x}_{\mathrm{k}} \\
\sum^{(2)} \mathrm{x}_{\mathrm{k}} \\
\vdots \\
\sum^{(\mathrm{n}+1)} \mathrm{x}_{\mathrm{k}}
\end{array}\right\}
\end{aligned}
$$

(3) can be expressed as

$$
c a=b
$$

It is easy to prove that $\mathrm{c}$ is a reversible square and that 
the equation (4) has a unique solution

$$
a=c^{-1} b
$$

in the equation - the $c^{-1}$ inverse matrix of square $c$. Substitute $a$ into the model formula (1) to get the prediction equation.

\section{Integrated energy system planning}

\subsection{RIES energy flow modeling}

The initial energy $P$ (electricity, gas, heat, light, wind, etc.) supplied to the region from the outside of the total energy flow of the region is converted, stored, transmitted, and managed on the demand side, which finally meets the end demand within the region $\mathrm{L}$. The process can be expressed as:

$$
L=f(\mathrm{P}, \mathrm{C}, \mathrm{N}, \mathrm{D})
$$

$\mathrm{C}, \mathrm{S}, \mathrm{N}$, and $\mathrm{D}$ represent the process of energy conversion, storage, transmission, and side management. Where $\mathrm{D}$ is related to the specific control strategy of demand-side management, it can be regarded as a modification of the end requirements, expressed as:

$$
L^{\prime}=D(L)
$$

$\mathrm{N}$ contains electricity, gas, heat (cold) and other system energy supply network transmission process, its characteristics vary, as discussed below.

1) Power system: Power system current calculation with node voltage amplitude and phase as the state variable, the establishment of active, reactive balance equation:

$$
\left\{\begin{array}{l}
P_{G i}-P_{L i}=U_{i} \sum_{i \in j} U_{j}\left(G_{i j} \cos \theta_{i j}+B_{i j} \sin \theta_{i j}\right. \\
Q_{G i}-Q_{L i}=U_{i} \sum_{i \in j} U_{j}\left(G_{i j} \sin \theta_{i j}+B_{i j} \cos \theta_{i j}\right.
\end{array}\right.
$$

Equation (8) has the following characteristics: $U_{i}=1$ $\theta_{i j}=0$

2) Natural gas system: natural gas system energy flow to node gas pressure as a state variable, the establishment of node gas balance equation:

$$
Q_{i}-\sum f_{i m}+\sum f_{n i}-\sum E_{i d} F_{d}=0
$$

In the model: $Q_{i}$ is the net injection of natural gas at node i, $f_{n i}$ and $f_{i m}$ are the injection of flow from upstream node $\mathrm{n}$ and the output flow to downstream node $\mathrm{m}$, respectively, $F_{d}$ is the gas consumption of the compressor, and the correlation coefficient $E_{i d}$ of one when the compressor extracts gas from node i, otherwise zero. Branch flow is calculated as follows:

$$
f_{i m}= \begin{cases}W_{i m} \sqrt{p_{i}^{2}-p_{m}^{2}} & p_{i} \geq p_{m} \\ -W_{i m} \sqrt{p_{m}^{2}-p_{i}^{2}} & p_{i}<p_{m}\end{cases}
$$

In the model: $f_{i m}$ is the flow of the branch $i m$,
$W_{i m}$ is the damping coefficient of the branch $i m, p_{i}$ and $p_{m}$ is the pressure of the node.

Compressor air consumption is:

$$
F_{d}=\left\{\begin{array}{l}
\frac{\alpha f_{i m}}{\eta_{d}}\left[k_{1}\left(\frac{p_{m}}{p_{i}}\right)^{\varphi}-k_{2}\right] \\
\frac{\alpha f_{i m}}{\eta_{d}}\left[k_{1}\left(\frac{p_{i}}{p_{m}}\right)^{\varphi}-k_{2}\right] p_{m} \geq p_{i} \\
p_{m}<p_{i}
\end{array}\right.
$$

For gas distribution networks without compressors, the type (9) can also be simplified to:

$$
p_{i}-p_{m}=s_{i m}\left|f_{i m}\right|^{\beta-1} f_{i m}
$$

In the equation: $\eta_{d}$ is compressor efficiency; $\alpha$, $\varphi, k_{1}, k_{2}$ is relevant constant coefficient, $S_{\text {im }}$ is pipe resistance coefficient, and $\beta$ is normal coefficient related to airflow and pipe characteristics.

3) Heating (cold) system: Heating (cold) system analysis includes two parts: hydro-analysis and thermal analysis. The hydraulic analysis selects the branch flow rate $M$ as the state variable. For a water supply network with $N$ nodes and $b$ branches can create $N-1$ flow balance equation and $b-N+1$ loop pressure drop equation can be established:

$$
\left\{\begin{array}{c}
A_{h} M=Q \\
B_{f}(S|M| M+Z-H)=0
\end{array}\right.
$$

In the equation: $A_{h}$ is the node-branch correlation matrix, $\mathrm{Q}$ is the net injection flow at each node, $B_{f}$ is the basic loop branch association matrix, $S$ is the pipe resistance coefficient verse array, $Z$ is the pipe elevation differential vector, $H$ is the branch water pump head vector. Thermal analysis establishes the temperature equilibrium equation of the water supply network and the return water network:

$$
\left\{\begin{array}{c}
A_{s} T_{s}=0 \\
A_{r} T_{r}+B_{r} T_{0}=0
\end{array}\right.
$$

In the system: $T_{s} T_{r}$ and $T_{0}$ are the water supply network, the return water network node and the user return water temperature vector, $A_{s} A_{r}$ and $B_{r}$ are the matrices related to flow, temperature and loss respectively.

4) Solution method: Although the above energy systems have different characteristics, they all satisfy Kirchhoff's law and the law of conservation of energy. The equations can be solved by the Newton-Raphson method or other iterative algorithms. The solution of the hybrid trend can be solved by constructing the integrated equation of the hybrid network, by using the unified iteration of the enumeration Jacobean matrix, or by allocating the energy equivalent of the energy coupling part to the separate solution methods of the mixed trend information to different energy sources. 


\subsection{RIES plans a commonality model}

Due to the close multi-energy coupling, the optimization space of RIES exists in the source, network, load, storage and other links. Early exploration of RIES planning at home and abroad focused on small energy supply systems consisting of a typical coupling device (CHP and CCHP, etc.) or a few identified devices, as well as the development of relevant operating strategies such as " power by heat ", without fully considering the optimization space of system equipment selection. Subsequent RIES planning is generally based on the EH theory, and through the over-layer modeling solution to achieve the device selection site selection and operation of the scheduling scheme of collaborative optimization. Combined with existing research results, the summary summarizes the decision variables that RIES can select, as shown in Table 1.

Table 1 RIES can select the decision variable

\begin{tabular}{|c|c|}
\hline Object. & The decision variable \\
\hline $\mathrm{EC}$ & $\begin{array}{c}\text { Configure } \mathrm{M}_{C} \text { capacity, site } \\
\text { selection, } \mathrm{S}_{C} \text { and scheduling } \\
\text { schemes } \mathrm{D}_{C}\end{array}$ \\
\hline ES & $\begin{array}{l}\text { Configure } \mathrm{M}_{S} \text { capacity, power, } \\
\mathrm{W}_{S} \text { location selection, and } \mathrm{S}_{C} \\
\text { scheduling schemes } \mathrm{D}_{C}\end{array}$ \\
\hline $\mathrm{EN}$ & $\begin{array}{c}\text { Pipeline selection, } \mathrm{T} \text { network } \\
\text { topology } \mathrm{N}\end{array}$ \\
\hline
\end{tabular}

This summarizes the regional IES planning commonality model as follows:

$$
\left\{\begin{array}{c}
\min f\left(\mathrm{M}_{C}, \mathrm{~S}_{C}, \mathrm{D}_{C}, \mathrm{M}_{S}, \mathrm{~W}_{S}, \mathrm{~S}_{S}, \mathrm{D}_{S}, \mathrm{~T}, \mathrm{~N}, \mathrm{D}_{L}\right) \\
\text { s.t.g }\left(\mathrm{M}_{C}, \mathrm{~S}_{C}, \mathrm{D}_{C}, \mathrm{M}_{S}, \mathrm{~W}_{S}, \mathrm{~S}_{S}, \mathrm{D}_{S}, \mathrm{~T}, \mathrm{~N}, \mathrm{D}_{L}\right)= \\
h\left(\mathrm{M}_{C}, \mathrm{~S}_{C}, \mathrm{D}_{C}, \mathrm{M}_{S}, \mathrm{~W}_{S}, \mathrm{~S}_{S}, \mathrm{D}_{S}, \mathrm{~T}, \mathrm{~N}, \mathrm{D}_{L}\right) \leq 0
\end{array}\right.
$$

In the series: $g$ and $h$ are the end sets of the equals and the sets of non-equal constraints in the planning model, respectively.

\section{Case validation}

There are many factors that affect the power load, such as weather, quarter, electricity price, politics, the location of the user, etc. This article uses the load data from July 1, 2019 to August 20,2019 (one month is counted as 30 days) in a certain area of Sichuan, china as the training data, sampling once a day, a total of 50 load data points; August 2019 the load from 21st to September 30th, 2019, a total of 40 points are used as test data. To verify the effectiveness of the planning scheme based on cumulative prediction, the two are compared, as shown in Figure1. As can be seen from Figure 1, the effect of the planning scheme based on the cumulative prediction method is significantly improved compared with that of the traditional planning scheme, and the prediction curve fits better, indicating that the optimization effect is significant and the prediction accuracy is higher.

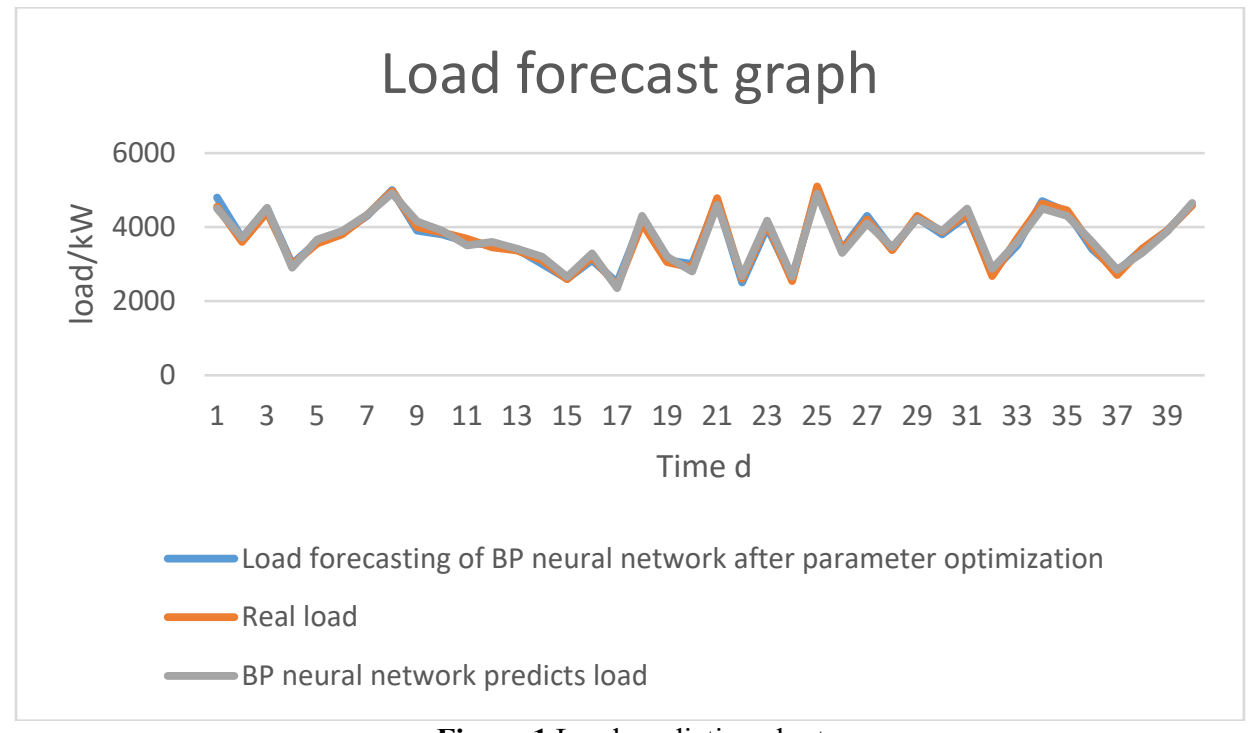

Figure 1 Load prediction chart

\section{Conclusions}

The regional integrated energy system takes the demandside multiple energy demand as the starting point, makes full use of the abundant renewable energy and unused energy in the region, and constructs the integrated energy supply system of source, network, lotus, storage interaction and coupling. Based on the basic concept of regional integrated energy system, this study comprehensively combs the current research status in this field by analyzing the key links in the process of planning design and evaluation, and puts forward the thinking of further development in the future. On the whole, the development of regional distributed energy system in the future must be planned first and supplemented by effective evaluation means. 


\section{Acknowledgment}

The authors would like to thank the support of the project

"Science and Technology Foundation of State Grid Corporation of China (5278991900MV)”.

\section{References}

1. ARANI L A, SADOUGHI F, LANGARIZADEH M. An expert system to diagnose pneumonia using fuzzy logic[J]. International Journal of Intelligent Systems, 2019, 27 (2) :103-107.

2. DONG Yuhong, FU Zetian, PENG Yaoqi, et al. Precision fertilization method of field crops based on the wavelet-BP neural network in China[J]. Journal of Cleaner Production, 2019, 27 (2) : 103-107.

3. YU Sheng, ZOU Hongbo, YU Fan, et al. Application of fuzzy neural network in power system short- term load forecasting[J]. Smart Grid, 2018, 46 (11):88-91, 97.

4. YUE Youjun, LIU Yinghan, ZHAO Hui, et al. Shortterm electric load forecasting based on CEEMDAN- SE and DBN[J/OL]. Electrical measurement and instrumentation. [2019- 1124].http://kns.cnki.net/kcms/detail/23.1202.TH.2019 0905.1700.004.html.

5. DONG Zihan. Regional power network load forecasting based on chaotic time series[J]. Power System and Clean Energy, 2019, 35 (5) :38-41.

6. ZHOU Xinghua, GENG Juncheng, DU Songhuai, et al. A summer short- term load forecasting method for temperature sensitive users considering demand response [J]. Power System and Clean Energy, 2019 , 35 (4) :16-22.

7. XU Qing, ZHOU Chao, ZHAO Shuangshuang, et al. Study on short-term electric load forecasting method based on machine learning[J/OL]. Electrical Measurement and Instrumentation. [2019-11-24]. http://kns.cnki.net/kcms/ detail/23.1202.TH.20191104.1614.018.html。

8. CHEN Linghui. Research on load balancing and decentralized dispatching method of renewable energy power system[J]. Power System and Clean Energy, 2019, 35 (12) : 60-66.

9. HU Wei, HU Yawei, YANG Feng.A wind power prediction neural network model based on improved flower pollination algorithm[J]. Journal of Systems Management, 2019, 28 (5) : 934-940.

10. TAO Zhiyong, CUI Xinxin. A hybrid improved flower pollination algorithm[J]. Transducer and Microsystems, 2019, 38 (10):139-142, 145. 